\title{
Emerging Role for Epithelial Polarity Proteins of the Crumbs Family as Potential Tumor Suppressors
}

\author{
Patrick Laprise \\ Department of Molecular Biology, Medical Biochemistry and Pathology/Cancer Research Center, \\ Laval University and CRCHUQ-Hôtel-Dieu de Québec, 9 McMahon, Québec, QC, Canada G1R 2J6 \\ Correspondence should be addressed to Patrick Laprise, patrick.laprise@crhdq.ulaval.ca
}

Received 30 May 2011; Accepted 9 July 2011

Academic Editor: Sanford I. Bernstein

Copyright (c) 2011 Patrick Laprise. This is an open access article distributed under the Creative Commons Attribution License, which permits unrestricted use, distribution, and reproduction in any medium, provided the original work is properly cited.

Defects in apical-basal polarity regulation are associated with tissue overgrowth and tumorogenesis, yet the molecular mechanisms linking epithelial polarity regulators to hyperplasia or neoplasia remain elusive. In addition, exploration of the expression and function of the full complement of proteins required for the polarized architecture of epithelial cells in the context of cancer is awaited. This paper provides an overview of recent studies performed on Drosophila and vertebrates showing that apical polarity proteins of the Crumbs family act to repress tissue growth and epithelial to mesenchymal transition. Thus, these proteins emerge as potential tumor suppressors. Interestingly, analysis of the molecular function of Crumbs proteins reveals a function for these polarity regulators in junctional complexes stability and control of signaling pathways regulating proliferation and apoptosis. Thereby, these studies provide a molecular basis explaining how regulation of epithelial polarity is coupled to tumorogenesis.

\section{Epithelial Polarity}

Epithelial tissues cover the surface and line internal cavities of the human body. Simple epithelia act as a diffusion barrier, generate vectorial transport, and sustain spatially oriented secretion to subdivide the body into morphologically and physiologically distinct compartments. The unidirectional nature of these functions requires the asymmetric distribution of many cellular constituents, a structural organization referred to as epithelial polarity. Epithelial polarization results from the regionalization of the plasma membrane into apical, lateral, and basal domains. The apical domain faces the external environment or a lumen, the lateral domain spans across the plane of the epithelium and contacts neighboring cells, and the basal domain is attached to the basement membrane (Figure 1). Apical junctional complexes are established at the interface between the apical and lateral domains to maintain the cohesion and impermeability of epithelia. Several proteins important for epithelial polarity have been identified in recent years, mainly in model organisms like C. elegans and Drosophila melanogaster [1]. The function of these proteins is conserved from worm to man, reflecting the significance of epithelial polarity. The importance of the polarized architecture of epithelial cells is further emphasized by the fact that numerous pathologies are associated with epithelial polarity defects, including most human cancers $[2,3]$.

\section{Roles of Crumbs Proteins in Epithelial Polarity Regulation}

Epithelial polarity is organized by a complex network of evolutionarily conserved proteins, including the apical transmembrane protein Crumbs (Crb) [1, 4-6]. Drosophila embryos lacking Crb display apical-basal polarity defects in several epithelia, which eventually collapse $[5,7]$. Overexpression of Crb dominantly extends the apical domain at the expense of the lateral domain [8], showing that Crb is an important apical determinant. Mutual antagonism between $\mathrm{Crb}$ and basolateral polarity modules is crucial for segregation and size control of membrane domains in epithelial cells, thus impacting on tissue morphogenesis [9$13]$.

The human genome encodes three Crb orthologs named CRB1, CRB2, and CRB3 [4]. CRB1 expression is restricted 

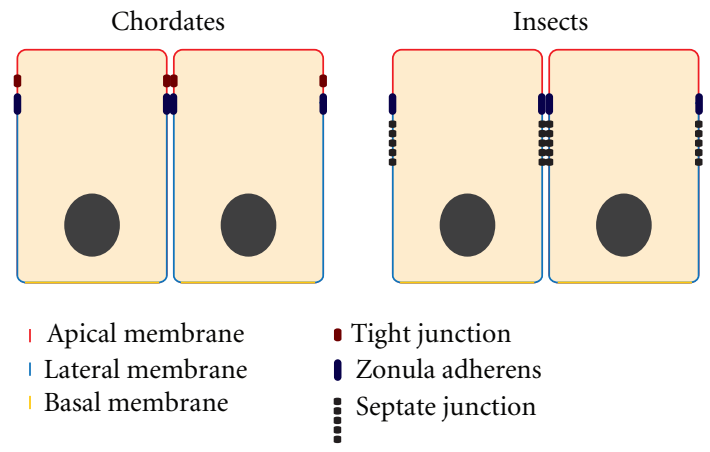

FIgURE 1: Organization of epithelial tissues. Epithelial cells are polarized along an apical-basal axis. The apical domain faces a lumen or the environment, the lateral domain contacts neighboring cells, and the basal domain is anchored to the basement membrane. This polarized architecture sustains unidirectional functions, such as vectorial transport. The apical and lateral domains are segregated by the zonula adherens, which is a circumferential adherens junction maintaining intercellular adhesion within epithelial tissues. In chordates epithelia, paracellular diffusion is limited by tight junctions, which sit apical to the zonula adherens. In insect, this sealing function is assumed by septate junctions that are established basal to the zonula adherens.

to the brain, cornea, and retina [14-16]. Mutations in human CRB1 or mouse Crb1 genes cause degenerative retinal pathologies [14, 17-19]. Similarly, loss of Crb is associated with light-induced retinal degeneration in flies [20]. CRB2 is expressed in several tissues [21], but its function remains poorly understood. CRB3 is expressed in most epithelia and exist as two splice variants. CRB3A is apically localized at the level of tight junctions $[4,22]$ and promotes intercellular junction formation as well as epithelial polarity [23-25]. CRB3B is associated with spindle poles in dividing cells or found in the apical cilium of polarized kidney epithelial cells to control cytokinesis and ciliogenesis, respectively [26].

Similar to Crb, CRB1 and CRB2 possess a large extracellular domain containing EFG and laminin repeats $[5,7$, 14]. In contrast, CRB3 has a short extracellular domain showing no clear homology with the other Crb proteins [22]. However, Drosophila Crb and the entire set of human CRB proteins contain a highly conserved cytoplasmic tail $[4,5]$, which is characterized by the presence of a FERM (4.1, ezrin, radixin, moesin) domain-binding site that is important for Crb function and regulation $[11,27,28]$. In addition, the last four amino acids (ERLI) of the cytoplasmic tail of Crb, CRB1, CRB2, and CRB3A interact with the protein Stardust (Sdt; named PALS1 in mammals) linking Crb or human CRB proteins to Drosophila PATJ (dPatj) or Patj, respectively [29-31]. Mutations in $s d t$ phenocopy $c r b$ loss-of-function $[29,32,33]$. In addition, the ability of exogenous Crb to rescue the $c r b$ mutant phenotype depends on the ERLI motif [27]. PALS1 has been implicated in epithelial polarity as well as cell-cell junction formation [25,34]. dPatj is important for Crb complex stabilization at the apical membrane, and Patj controls the delivery of CRB3 to the apical domain as well as tight junction formation [35-37]. These studies suggest that
Sdt/PALS1 and dPatj/Patj play crucial roles as downstream effectors and/or regulators of Crb/CRB3.

\section{Polarity and Cancer}

Loss of cell polarity is a typical hallmark of tumor progression in epithelial tissues. The polarized architecture of epithelial cells is compromised at early steps of epithelial to mesenchymal transition (EMT), a process also associated with loss of cell-cell adhesion and acquisition of migratory and invasive properties [38]. Thus, EMT is a critical step in carcinoma progression and metastasis. These observations predict that regulators of apical-basal polarity are fundamental to preserve epithelial homeostasis and to limit tumorogenesis. This hypothesis was first supported by studies in Drosophila showing that loss of any members of the lateral-promoting Scribble (Scrib) polarity module promotes epithelial tissue overgrowth and disorganization, resulting in a tumor-like phenotype [39]. The tumor suppressor function of Scrib is conserved in mice [40]. These data have inspired a number of scientists who demonstrated that the expression of many epithelial polarity regulators is altered in several cancers and that proteins required for epithelial polarization are important targets of viral oncoproteins $[2,3,41]$. Finally, mutations in the gene encoding the polarity protein Lkb1 cause a genetic syndrome associated with a high incidence of cancer [42]. Together, these recent discoveries clearly established that further characterization of proteins coordinating epithelial polarity will contribute to our understanding of cancer biology. Deciphering the molecular mechanisms by which polarity proteins act as tumor suppressors is a major issue yet to be solved in this field of research.

3.1. CRB3 and Tumor Growth. The role of the polarity protein CRB3 in cancer was not thoroughly studied yet, but increasing evidence suggests that this protein could restrict tumor progression. Gene expression profiling revealed that repression of CRB3 expression correlates with increased tumorigenic potential in mouse kidney epithelial cells. Reexpression of CRB3 restored cell-cell junctions integrity and cell polarization, while limiting cell motility and metastasis [43]. This suggests that loss of CRB3 in tumor cells is not coincidental, but plays an active role in tumorogenesis. A mechanism leading to the loss of CRB3 expression was recently elucidated. Indeed, CRB3 expression is repressed by two factors promoting EMT, namely, the transcription regulators Snail and ZEB1 [44-46]. Expression of these proteins alters cell-cell adhesion, while increasing migration, invasion, and metastasis $[45,47,48]$. Snail and ZEB1 directly bind to and repress CRB3 promoter $[44,46]$. Importantly, expression of exogenous CRB3 in Snail-expressing cells partially restores the formation of cell-cell junctions and the epithelial phenotype, suggesting that CRB3 gene is a functional target of Snail and that its repression contributes to EMT [46]. Expression of Snail and ZEB1 is increased in many human tumors, and it correlates with dedifferentiation and invasion [47-49]. This suggests that CRB3 expression is 
reduced in human cancers allowing for tumor progression, but a former demonstration of this hypothesis remains awaited. Collectively, these findings establish that it is of great interest to study CRB3 in tumor of epithelial origin, which accounts for the vast majority of human cancers.

\subsection{Mechanisms by Which Crb Proteins Could Act as Tumor Suppressors}

3.2.1. Crb and CRB3 Activates the Tumor Suppressor Salvador/Warts/Hippo Pathway. Although it is clear that alteration of epithelial polarity contributes to tissue overgrowth, mechanistic insights into how polarity regulators act in this context are missing. Recent studies on Drosophila and mammalian cells have shown that $\mathrm{Crb}$ and CRB3 regulate signaling pathways controlling proliferation and survival, including the Salvador/Warts/Hippo (SWH) pathway. In Drosophila, the adaptor protein Salvador (Sav) associates with the kinase Hippo (Hpo), which phosphorylates and activates the kinase Warts (Wts) [50]. Activated Wts, in association with mob as a tumor suppressor (Mats), phosphorylates the transcriptional coactivator Yorkie (Yki). Phospho-Yki is sequestered in the cytoplasm and unable to activate its proproliferative (cyclin E, E2F1, and bantam) and antiapoptotic (Drosophila inhibitor of apoptosis (diap1)) target genes [50, 51]. Thus, inhibition of the SWH pathway leads to Yki-dependent stimulation of cell proliferation and survival, leading to tissue hyperplasia (Figure 2) [51]. The cortical FERM-domain protein Expanded (Ex) also represses Yki-dependent transcription. Ex may act by binding directly to Yki to limit its nuclear translocation [52]. Proper localization at apical junctions and function of Ex depend on the protocadherin Fat, which potentially couples extracellular signals to Yki regulation [53-55]. The SWH pathway is well conserved in mammals and contributes to cell growth regulation. Overexpression of the Yki ortholog YAP promotes EMT and uncontrolled proliferation [56]. A similar scenario prevails in vivo where YAP overexpression increases liver size and expands progenitor cell population in the intestinal epithelium $[57,58]$. Based on these studies, it is not surprising that LATS1, MOB, and SAV (the homolog of Wts, Mats and Sav, resp.) were described as tumor suppressors [59-62]. Thus, further defining the regulation of the SWH pathway will refine our understanding of tissue growth and organ size regulation, as well as human diseases associated with proliferation and apoptosis deregulation such as cancer. In particular, identification of upstream regulators of the SWH and determination of their mechanism of action represent an important challenge.

Loss of $\mathrm{crb}$ leads to tissue hyperplasia in Drosophila larval epithelial discs [63-65]. Reduction of Crb amount increases organ overgrowth resulting from Yki overexpression, and a $\mathrm{crb}$ null allele enhances tissue enlargement in a hypomorphic ex background [64, 66]. Moreover, lack of Crb increases the expression of Yki target genes [64]. This suggests that $\mathrm{Crb}$ is a positive regulator of Ex and SWH pathway and contributes to limiting Yki activity. In the absence of Crb, Ex protein accumulates, but is delocalized basally to its normal location $[63,64,66]$. This suggests that Crb-dependent Ex localization is crucial for its function. The recruitment of Ex by Crb likely depends on a direct binding of these proteins [63]. Whether there is collaboration between Fat and Crb to localize Ex remains to be determined [53, 54]. Intriguingly, overexpression of $\mathrm{Crb}$ or expression of the membrane-bound cytoplasmic tail of $\mathrm{Crb}\left(\mathrm{Crb}_{\text {intra }}\right)$ decreases Ex levels and increases cell growth through Yki activation [63, 64, 66-68]. Thus, lack of Crb or excessive amount of this protein results in a similar Yki-dependent phenotype. This could suggests that $\mathrm{Crb}$ overabundance has a dominant negative effect, perhaps by disrupting stoichiometry of a Crb-containing complex. However, the opposite effect of $\mathrm{Crb}_{\text {intra }}$ expression or loss of Crb on Ex levels argues against this hypothesis and rather suggest that $\mathrm{Crb}$ has a complex function within the SWH pathway. One plausible hypothesis is that Crb plays an important role in fine-tuning of the SWH pathway activity by favoring Ex function through an effect on its localization, while limiting its level (Figure 2). Collectively, these data show that Crb is a regulator of the SWH pathway, conferring to $\mathrm{Crb}$ the ability to control proliferation and epithelial tissue size.

In mammals, high cell density leads to a cytoplasmic retention of YAP through the activation of the SWH pathway, which thereby contributes to contact-mediated inhibition of growth $[68,69]$. This implies that a receptor sensing cell density relays information to the SWH pathway to limit proliferation. Similar to YAP, members of the SMAD family, which regulate transcription downstream of TGF- $\beta$ signaling [70], are enriched in the nucleus of cells cultivated at low density, whereas they are trapped in the cytoplasm of epithelial cells grown at high density [71]. Interestingly, phosphorylated YAP binds to SMAD2/3, suggesting that phospho-YAP could contribute to the cytoplasmic retention of these proteins. Accordingly, knockdown of LATS kinases results in a nuclear accumulation of both YAP and SMAD2/3 along with an increase in expression of TGF- $\beta$ target genes [71]. This shows that the SWH pathway regulates TGF$\beta /$ SMADs signaling through a YAP-dependent regulation of SMAD localization. Strikingly, YAP binds to CRB3 complex components, and knockdown of CRB3 decreases YAP phosphorylation. Reduction of CRB3 correlates with YAP and SMAD2/3 nuclear accumulation and potentiates TGF$\beta$-induced EMT [71]. This mechanism may explain how the loss of CRB3 leads to EMT in other cell types [46]. Thus, like Drosophila Crb, CRB3 participates in the regulation of the SWH pathway. In particular, CRB3 couples cell density to activation of this pathway. It will be interesting to investigate whether this functional relationship has a tumor suppressor function in humans. One initial step would be to correlate CRB3 expression and localization with Yki target genes' expression in cancer tissues.

3.2.2. Control of Notch Signaling by Crb. Notch proteins are evolutionarily conserved transmembrane receptors, which are activated by transmembrane ligands expressed at the surface of adjacent cells [72]. Notch-mediated short-range intercellular communication fulfills crucial roles in embryonic development and tissue renewal. Notch activation 


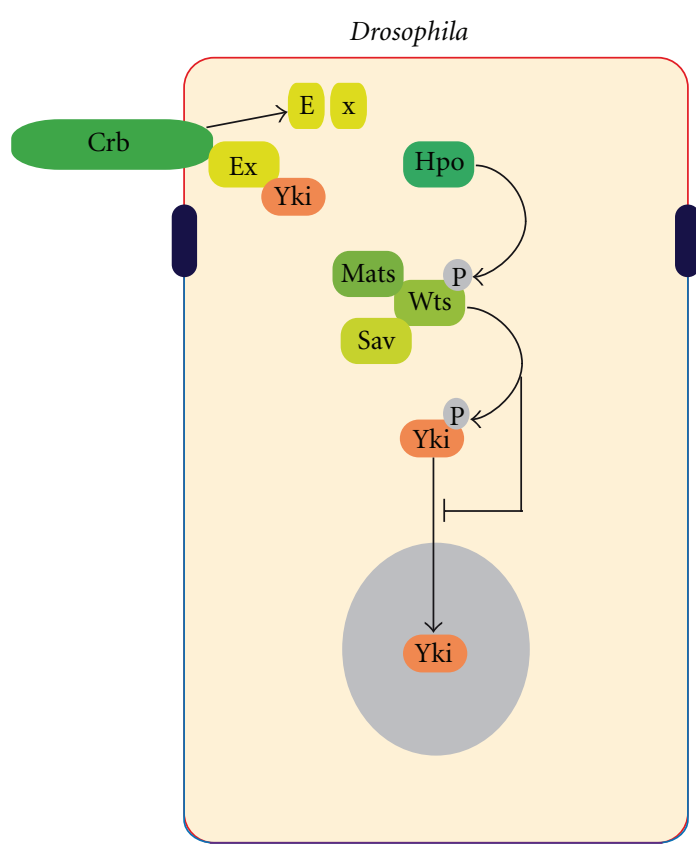

(a)

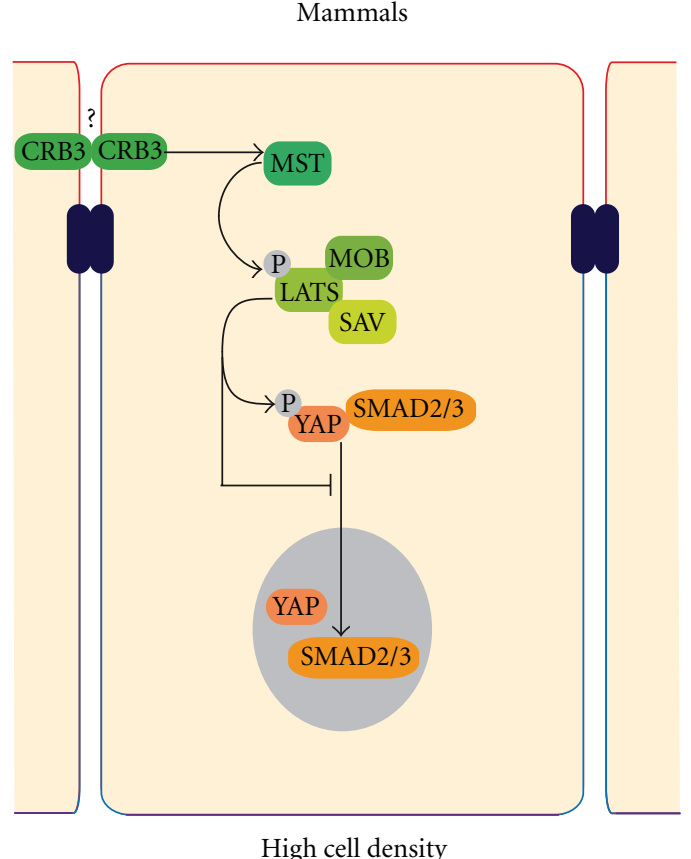

(b)

\begin{tabular}{|lll|}
\hline \multicolumn{2}{|c|}{ Conserved Salvador-Warts-Hippo pathway components } \\
\hline Prosophila & Mammals & Protein family/function \\
\hline Hpo & YAP & Transcriptional coactivators \\
Wts & MST1/2 & Sterile 20-like kinases \\
Mats & LATS1/2 & DBF family kinases \\
Sav & MOB & Adaptor proteins \\
\hline
\end{tabular}

Figure 2: Functional relationship linking Crb proteins to the SWH pathway. (a) In Drosophila, nuclear Yki promotes cell proliferation and survival by modulating gene expression. Activation of the SWH pathway leads to the phosphorylation and cytoplasmic retention of Yki. Binding to the FERM domain protein Ex also prevents the nuclear translocation of Yki. Crb restricts Yki-dependent tissue hyperplasia by contributing to Ex localization and function. Overexpression of Crb leads to degradation of Ex, suggesting that Crb plays a crucial role in the fine-tuning of the SWH activity by activating Ex, while limiting its level. The table lists the components of the SWH pathway in Drosophila and mammals. (b) In mammalian cells, high cell density activates the SWH pathway, which contributes to contact-mediated inhibition of growth. Phosphorylation of YAP also results in cytoplasmic sequestration of SMAD proteins, thus limiting TGF- $\beta$ responsiveness. CRB3 is important to couple cell density information to the SWH, perhaps through a homophilic interaction, leading to the suppression of TGF- $\beta$ signaling and epithelial to mesenchymal transition.

influences cell fate specification, proliferation, apoptosis, and differentiation. It is therefore not surprising that deregulation of Notch signaling has profound effects on tissue homeostasis and results in human pathologies, including many human cancers [73]. Following ligand binding, Notch intracellular domain (NICD) is released by proteolysis. Full proteolytic processing of NICD requires the multimeric $\gamma$-secretase complex. Processed NICD reaches the nucleus where it partakes in a transcription complex allowing for expression of Notch pathway target genes [72].

In Drosophila wing epithelial discs, Notch promotes the expression of Crb, which represses Notch activity [74]. Thus, Notch signaling refines itself by inducing the expression of its repressor. The function of Crb in limiting Notch signaling is not restricted to the wing disc epithelium. Loss of Crb in the developing eye imaginal disc results in Notch-dependent 
overproliferation and tissue hyperplasia [65]. Several mechanisms may explain the effect of Crb on Notch signaling. First, Crb inhibits endocytosis of Notch [65], which is required for activation of Notch signaling $[75,76]$. Secondly, Crb limits $\gamma$-secretase complex activity, thus interfering with Notch processing [74]. Similarly, human CRB2 inhibits $\gamma$-secretase action in cell lines and cell-free assays [77]. CRB1 and CRB3 also show a similar inhibitory effect. CRB2 associates with components of the $\gamma$-secretase complex. This interaction depends on CRB2 transmembrane domain, which is essential for $\gamma$-secretase complex inhibition [77]. This suggests that CRB2 counteracts $\gamma$-secretase-mediated proteolysis through a direct interaction. Crb family proteins are also functionally linked to Notch signaling in zebra fish. In this vertebrate organism, the extracellular domain of Crb binds to Notch and inhibits its activity in cis [78]. This mechanism involving a direct interaction of $\mathrm{Crb}$ with Notch might be conserved, as Crb-dependent Notch inhibition in flies requires the extracellular domain of Crb [65]. Finally, the Crb-dependent regulation of Notch may depend on the ability of $\mathrm{Crb}$ to activate the SWH pathway. Indeed, YAP1 stimulates proliferation through activation of Notch signaling [58]. In addition, the membrane localization of Notch is increased in hpo mutant clones [79]. However, the SWH pathway, and Notch can activate each other in Drosophila ovaries $[80,81]$. Thus, more work is required at this point to clarify the complex functional relationship linking Crb, the SWH pathway and Notch signaling. Overall, these studies establish that $\mathrm{Crb}$ proteins share an evolutionarily conserved function in limiting Notch signaling. Several mechanisms have been proposed to explain how Crb family members limit Notchmodulated cell behavior (Figure 3), but further studies are required to clarify whether lack of CRB proteins favor tumor growth through activation of Notch signaling.

\subsubsection{Regulation of Apical Junctional Complexes by Crb} Proteins. The barrier function of epithelia relies on different types of junctional complexes, which maintain cohesion between epithelial cells and seal the intercellular space. The zonula adherens (ZA), a belt-like adherens junction, makes prominent contribution to adhesive forces holding epithelial cells together [82]. The ZA also plays crucial roles in tissue morphogenesis and homeostasis. The homophilic adhesion receptor E-cadherin is a core component of the ZA. E-cadherin indirectly links the cortical microfilaments of neighboring cells through cytoplasmic adaptor proteins, including $\beta$-catenin [82]. Drosophila $\mathrm{Crb}$ is required for proper assembly of the ZA in epithelial and photoreceptor cells $[83,84]$. Moreover, Crb contributes to precisely localizing the ZA through apical displacement of the scaffold protein Bazooka (Baz; Par3 in c. elegans and vertebrates), which acts as a landmark to establish the ZA at the apicallateral border [85-88]. The capacity of Crb to promote ZA integrity may confer to this protein the ability to maintain epithelial tissue homeostasis. Indeed, E-cadherin has an important tumor suppressor function, by limiting proliferation, invasion, and metastasis [89]. Many human tumor types show a loss of E-cadherin expression. Loss of

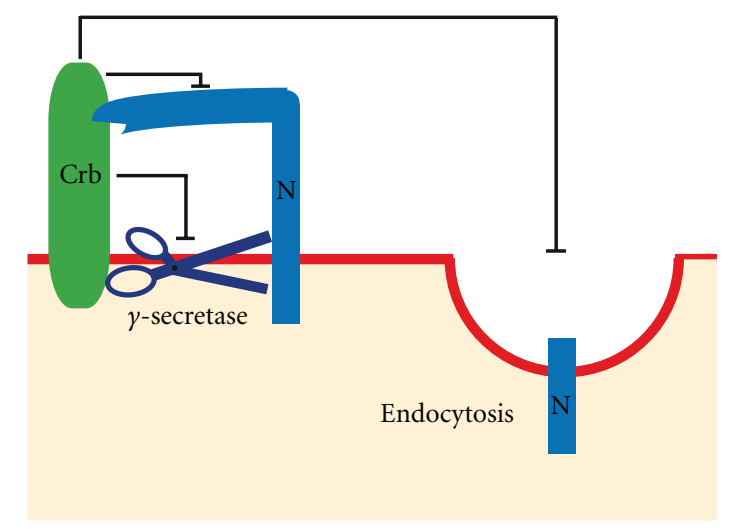

Figure 3: Crb proteins control Notch-dependent tissue growth. Crb proteins repress Notch $(\mathrm{N})$ signaling in Drosophila, zebra fish, and mammalian cells. Different mechanisms account for the Crb-dependent inhibition of Notch signaling. Drosophila Crb and CRB2 inhibit $\gamma$-secretase activity. In addition, Crb limits Notch endocytosis, which is important for Notch signaling. Finally, the extracellular domain of Crb proteins binds to Notch and counteracts its activity.

E-cadherin expression in cancer may be explained by loss of heterozygosity, epigenetic modification of the E-cadherin locus, and transcriptional silencing by EMT-promoting factors. Many mechanisms other than genetic alterations or epigenetic modifications can interfere with E-cadherin function. For instance, regulation of E-cadherin level at the plasma membrane by endocytosis is important for ZA integrity [89]. Interestingly, appropriate level of Crb resulting from the equilibrium between its delivery, endocytosis, and recycling could play an important role in stabilizing Ecadherin at the membrane, [90, 91]. However, a formal demonstration of this hypothesis is awaited. In addition, expression of E-cadherin is strongly repressed during EMT [38], which could be repressed by CRB3. Thus, it would be pertinent to investigate whether there is a correlation between CRB proteins and E-cadherin expression in human cancers and to better define CRB proteins function in the regulation of ZA integrity, especially in vertebrates.

While the cohesion of epithelial cells largely depends on the ZA, the selective permeability of epithelia relies on tight junctions (TJs) in vertebrates and septate junctions in invertebrates [92, 93]. TJs sit just apical to the ZA and restrict paracellular diffusion. Several transmembrane proteins are associated with TJ, including members of the claudin family, which are core components of TJ required for their assembly, and CRB3 $[94,95]$. In the absence of CRB3, TJ integrity is compromised [95]. Structure-function analysis revealed that the C-terminal ERLI motif is required for the positive impact of CRB3 on TJ organization [43, 95]. Consistent with this observation, PALS1 and Patj, which are recruited into the CRB3 complex through the ERLI domain, are also required to establish functional TJ $[34,35]$. Dysfunctions of TJ were linked to cancer over recent years [94]. Overexpression of $\mathrm{ZO}-1$, a protein associated with the cytoplasmic face of TJ, blocks proliferation in cultured epithelial cells [96]. 
Thus, it is not surprising that ZO-1 level is decreased in several human tumors $[97,98]$. The mechanism used by ZO-1 or other TJ proteins to maintain a normal epithelial phenotype remains elusive. However, increasing evidence suggests that TJ proteins have an impact on proliferation through regulation of gene expression. For instance, ZO-1 binds to and sequesters the dual-location protein ZONAB at TJ $[96,99]$. Loss of ZO-1 or disruption of TJ releases this protein, which can then translocate to the nucleus where it acts as a transcription factor. ZONAB upregulates the expression of cell cycle regulators, DNA replication factors, and oncogenes [96, 99, 100]. Moreover, ZONAB binds to CDK4 to favor its nuclear accumulation, thus facilitating the G1/S transition [96]. ZO-2, a TJ-associated protein related to ZO-1, can also regulate gene expression. ZO-2 can shuttle in the nucleus to repress cyclin D1 expression [101]. Thus, it is possible that CRB3 limits proliferation by maintaining TJ integrity and sustaining TJ-dependent gene expression regulation. However, it will be important to investigate whether CRB3 has a direct impact on the regulation of TJassociated transcription factor regulating proliferation such as ZONAB.

\section{Conclusions and Perspectives}

Recent studies have established that Crb/CRB proteins regulate epithelial tissue growth by acting as transmembrane proteins controlling intracellular signaling important for proliferation and apoptosis. Crb/CRB proteins are linked to many pathways through different domains, showing a complex function for these proteins in relaying growthcontrol signals. Indeed, Crb/CRBs inhibit Notch signaling through a direct interaction with the extracellular domain of Notch receptor in cis, counteract $\gamma$-secretase activity using the transmembrane domain, control Ex localization activity using the FERM domain-binding site within the cytoplasmic tail, while promoting $\mathrm{TJ}$ integrity through the Cterminal ERLI amino acids. These studies therefore provide mechanistic insights linking a cell polarity regulator to restriction of tissue hyperplasia. One outstanding question is whether CRB3 acts as a receptor transmitting extracellular cues inside the cell to maintain epithelial homeostasis. Although the extracellular domain of CRB3 is short, it may be involved in protein-protein interaction or may bind to lectin proteins, as it is glycosylated [22]. Identification of binding partners for the extracellular domain of CRB3 would help in addressing this question. Further investigation is required at this point to formerly establish that CRB3 is a tumor suppressor in humans, but deciphering the molecular mechanisms acting downstream of CRB3 seems a promising avenue to better understand cancer biology and to identify potential therapeutic targets.

\section{Acknowledgment}

The Canadian Cancer Society Research Institute funds research on epithelial polarity in P. L. laboratory.

\section{References}

[1] P. Laprise and U. Tepass, "Novel insights into epithelial polarity proteins in Drosophila," Trends in Cell Biology, vol. 21, no. 7, pp. 401-408, 2011.

[2] L. E. Dow and P. O. Humbert, "Polarity regulators and the control of epithelial architecture, cell migration, and tumorigenesis," International Review of Cytology, vol. 262, pp. 253-302, 2007.

[3] D. Coradini, C. Casarsa, and S. Oriana, "Epithelial cell polarity and tumorigenesis: new perspectives for cancer detection and treatment," Acta Pharmacologica Sinica, vol. 32, no. 5, pp. 552-564, 2011.

[4] E. Bazellieres, E. Assemat, J. P. Arsanto, A. Le Bivic, and D. Massey-Harroche, "Crumbs proteins in epithelial morphogenesis," Frontiers in Bioscience, vol. 14, pp. 2149-2169, 2009.

[5] N. A. Bulgakova and E. Knust, "The Crumbs complex: from epithelial-cell polarity to retinal degeneration," Journal of Cell Science, vol. 122, no. 15, pp. 2587-2596, 2009.

[6] J. Pieczynski and B. Margolis, "Protein complexes that control renal epithelial polarity," The American Journal of Physiology-Renal Physiology, vol. 300, no. 3, pp. F589-F601, 2011.

[7] U. Tepass, C. Theres, and E. Knust, "crumbs encodes an EGFlike protein expressed on apical membranes of Drosophila epithelial cells and required for organization of epithelia," Cell, vol. 61, no. 5, pp. 787-799, 1990.

[8] A. Wodarz, U. Hinz, M. Engelbert, and E. Knust, "Expression of crumbs confers apical character on plasma membrane domains of ectodermal epithelia of Drosophila," Cell, vol. 82, no. 1, pp. 67-76, 1995.

[9] G. Tanentzapf and U. Tepass, "Interactions between the crumbs, lethal giant larvae and bazooka pathways in epithelial polarization," Nature Cell Biology, vol. 5, no. 1, pp. 46-52, 2003.

[10] D. Bilder, M. Schober, and N. Perrimon, "Integrated activity of PDZ protein complexes regulates epithelial polarity," Nature Cell Biology, vol. 5, no. 1, pp. 53-58, 2003.

[11] P. Laprise, S. Beronja, N. F. Silva-Gagliardi et al., "The FERM protein Yurt is a negative regulatory component of the Crumbs complex that controls epithelial polarity and apical membrane size," Developmental Cell, vol. 11, no. 3, pp. 363374, 2006.

[12] P. Laprise, K. M. Lau, K. P. Harris et al., "Yurt, Coracle, Neurexin IV and the $\mathrm{Na}(+), \mathrm{K}(+)$-ATPase form a novel group of epithelial polarity proteins," Nature, vol. 459, no. 7250, pp. 1141-1145, 2009.

[13] P. Laprise, S. M. Paul, J. Boulanger, R. M. Robbins, G. J. Beitel, and U. Tepass, "Epithelial polarity proteins regulate Drosophila tracheal tube size in parallel to the luminal matrix pathway," Current Biology, vol. 20, no. 1, pp. 55-61, 2010.

[14] A. I. den Hollander, J. B. ten Brink, Y. J. M. de Kok et al., "Mutations in a human homologue of Drosophila crumbs cause retinitis pigmentosa (RP12)," Nature Genetics, vol. 23, no. 2, pp. 217-221, 1999.

[15] A. I. den Hollander, M. Ghiani, Y. J. M. de Kok et al., "Isolation of Crb1, a mouse homologue of Drosophila crumbs, and analysis of its expression pattern in eye and brain," Mechanisms of Development, vol. 110, no. 1-2, pp. 203-207, 2001.

[16] J. Beyer, X. C. Zhao, R. Yee et al., "The role of crumbs genes in the vertebrate cornea," Investigative Ophthalmology \& Visual Science, vol. 51, no. 9, pp. 4549-4556, 2010. 
[17] A. K. Mehalow, S. Kameya, R. S. Smith et al., "CRB1 is essential for external limiting membrane integrity and photoreceptor morphogenesis in the mammalian retina," Human Molecular Genetics, vol. 12, no. 17, pp. 2179-2189, 2003.

[18] S. A. van de Pavert, J. Meuleman, A. Malysheva et al., "A single amino acid substitution (Cys249Trp) in Crb1 causes retinal degeneration and deregulates expression of pituitary tumor transforming gene Pttg1," Journal of Neuroscience, vol. 27, no. 3, pp. 564-573, 2007.

[19] M. Richard, R. Roepman, W. M. Aartsen et al., "Towards understanding CRUMBS function in retinal dystrophies," Human Molecular Genetics, vol. 15, no. 2, pp. R235-R243, 2006.

[20] K. Johnson, F. Grawe, N. Grzeschik, and E. Knust, “Drosophila crumbs is required to inhibit light-induced photoreceptor degeneration," Current Biology, vol. 12, no. 19, pp. 16751680, 2002.

[21] J. A. van den Hurk, P. Rashbass, R. Roepman et al., "Characterization of the Crumbs homolog 2 (CRB2) gene and analysis of its role in retinitis pigmentosa and Leber congenital amaurosis," Molecular Vision, vol. 11, pp. 263-273, 2005.

[22] O. Makarova, M. H. Roh, C. J. Liu, S. Laurinec, and B. Margolis, "Mammalian Crumbs3 is a small transmembrane protein linked to protein associated with Lin-7 (Pals1)," Gene, vol. 302, no. 1-2, pp. 21-29, 2003.

[23] C. Lemmers, D. Michel, L. Lane-Guermonprez et al., "CRB3 binds directly to Par6 and regulates the morphogenesis of the tight junctions in mammalian epithelial cells," Molecular Biology of the Cell, vol. 15, no. 3, pp. 1324-1333, 2004.

[24] T. W. Hurd, L. Gao, M. H. Roh, I. G. Macara, and B. Margolis, "Direct interaction of two polarity complexes implicated in epthelial tight junction assembly," Nature Cell Biology, vol. 5, no. 2, pp. 137-142, 2003.

[25] M. H. Roh, S. Fan, C. J. Liu, and B. Margolis, "The Crumbs3Pals1 complex participates in the establishment of polarity in mammalian epithelial cells," Journal of Cell Science, vol. 116, no. 14, pp. 2895-2906, 2003.

[26] S. Fan, V. Fogg, Q. Wang, X. W. Chen, C. J. Liu, and B. Margolis, "A novel Crumbs3 isoform regulates cell division and ciliogenesis via importin beta interactions," Journal of Cell Biology, vol. 178, no. 3, pp. 387-398, 2007.

[27] A. Klebes and E. Knust, "A conserved motif in Crumbs is required for E-cadherin localisation and zonula adherens formation in Drosophila," Current Biology, vol. 10, no. 2, pp. 76-85, 2000.

[28] E. Médina, J. Williams, E. Klipfell, D. Zarnescu, G. Thomas, and A. L. Bivic, "Crumbs interacts with moesin and beta(Heavy)-spectrin in the apical membrane skeleton of Drosophila," Journal of Cell Biology, vol. 158, no. 5, pp. 941951, 2002.

[29] A. Bachmann, M. Schneider, E. Theilenberg, F. Grawe, and E. Knust, "Drosophila Stardust is a partner of Crumbs in the control of epithelial cell polarity," Nature, vol. 414, no. 6864, pp. 638-643, 2001.

[30] N. A. Bulgakova, O. Kempkens, and E. Knust, "Multiple domains of Stardust differentially mediate localisation of the Crumbs-Stardust complex during photoreceptor development in Drosophila," Journal of Cell Science, vol. 121, no. 12, pp. 2018-2026, 2008.

[31] M. H. Roh, O. Makarova, C. J. Liu et al., "The Maguk protein, Pals1, functions as an adapter, linking mammalian homologues of Crumbs and Discs Lost," Journal of Cell Biology, vol. 157, no. 1, pp. 161-172, 2002.
[32] U. Tepass and E. Knust, "Crumbs and stardust act in a genetic pathway that controls the organization of epithelia in Drosophila melanogaster," Developmental Biology, vol. 159, no. 1, pp. 311-326, 1993.

[33] Y. Hong, B. Stronach, N. Perrimon, L. Y. Jan, and Y. N. Jan, "Drosophila Stardust interacts with Crumbs to control polarity of epithelia but not neuroblasts," Nature, vol. 414, no. 6864, pp. 634-638, 2001.

[34] S. W. Straight, K. Shin, V. C. Fogg et al., "Loss of PALS1 expression leads to tight junction and polarity defects," Molecular Biology of the Cell, vol. 15, no. 4, pp. 1981-1990, 2004.

[35] D. Michel, J. P. Arsanto, D. Massey-Harroche, C. Béclin, J. Wijnholds, and A. Le Bivic, "PATJ connects and stabilizes apical and lateral components of tight junctions in human intestinal cells," Journal of Cell Science, vol. 118, no. 17, pp. 4049-4057, 2005.

[36] M. Richard, F. Grawe, and E. Knust, "DPATJ plays a role in retinal morphogenesis and protects against light-dependent degeneration of photoreceptor cells in the Drosophila eye," Developmental Dynamics, vol. 235, no. 4, pp. 895-907, 2006.

[37] K. Shin, S. Straight, and B. Margolis, "PATJ regulates tight junction formation and polarity in mammalian epithelial cells," Journal of Cell Biology, vol. 168, no. 5, pp. 705-711, 2005.

[38] J. P. Thiery, H. Acloque, R. Y. Huang, and M. A. Nieto, "Epithelial-mesenchymal transitions in development and disease," Cell, vol. 139, no. 5, pp. 871-890, 2009.

[39] D. Bilder, "Epithelial polarity and proliferation control: links from the Drosophila neoplastictumor suppressors," Genes and Development, vol. 18, no. 16, pp. 1909-1925, 2004.

[40] L. Zhan, A. Rosenberg, K. C. Bergami et al., "Deregulation of scribble promotes mammary tumorigenesis and reveals a role for cell polarity in carcinoma," Cell, vol. 135, no. 5, pp. 865-878, 2008.

[41] L. Huang and S. K. Muthuswamy, "Polarity protein alterations in carcinoma: a focus on emerging roles for polarity regulators," Current Opinion in Genetics and Development, vol. 20, no. 1, pp. 41-50, 2010.

[42] J. L. Herrmann, Y. Byekova, C. A. Elmets, and M. Athar, "Liver Kinase B1 (LKB1) in the pathogenesis of epithelial cancers," Cancer Letters, vol. 306, no. 1, pp. 1-9, 2011.

[43] C. M. Karp, T. T. Ting, R. Mathew et al., "Role of the polarity determinant crumbs in suppressing mammalian epithelial tumor progression," Cancer Research, vol. 68, no. 11, pp. 4105-4115, 2008.

[44] K. Aigner, B. Dampier, L. Descovich et al., "The transcription factor ZEB1 (deltaEF1) promotes tumour cell dedifferentiation by repressing master regulators of epithelial polarity," Oncogene, vol. 26, no. 49, pp. 6979-6988, 2007.

[45] S. Spaderna, O. Schmalhofer, M. Wahlbuhl et al., "The transcriptional repressor ZEB1 promotes metastasis and loss of cell polarity in cancer," Cancer Research, vol. 68, no. 2, pp. 537-544, 2008.

[46] E. L. Whiteman, C. J. Liu, E. R. Fearon, and B. Margolis, "The transcription factor snail represses Crumbs 3 expression and disrupts apico-basal polarity complexes," Oncogene, vol. 27, no. 27, pp. 3875-3879, 2008.

[47] G. Browne, A. E. Sayan, and E. Tulchinsky, “ZEB proteins link cell motility with cell cycle control and cell survival in cancer," Cell Cycle, vol. 9, no. 5, pp. 886-891, 2010.

[48] Y. Wu and B. P. Zhou, "Snail: more than EMT," Cell Adhesion and Migration, vol. 4, no. 2, pp. 199-203, 2010. 
[49] Y. Wu and B. P. Zhou, "TNF-alpha/NF-kappaB/Snail pathway in cancer cell migration and invasion," The British Journal of Cancer, vol. 102, no. 4, pp. 639-644, 2010.

[50] A. Genevet and N. Tapon, "The Hippo pathway and apicobasal cell polarity," The Biochemical Journal, vol. 436, no. 2, pp. 213-224, 2011.

[51] C. Badouel, A. Garg, and H. McNeill, "Herding Hippos: regulating growth in flies and man," Current Opinion in Cell Biology, vol. 21, no. 6, pp. 837-843, 2009.

[52] C. Badouel, L. Gardano, N. Amin et al., "The FERMdomain protein Expanded regulates Hippo pathway activity via direct interactions with the transcriptional activator Yorkie," Developmental Cell, vol. 16, no. 3, pp. 411-420, 2009.

[53] F. C. Bennett and K. F. Harvey, "Fat cadherin modulates organ size in Drosophila via the Salvador/Warts/Hippo signaling pathway," Current Biology, vol. 16, no. 21, pp. 21012110, 2006.

[54] E. Silva, Y. Tsatskis, L. Gardano, N. Tapon, and H. McNeill, "The tumor-suppressor gene fat controls tissue growth upstream of expanded in the hippo signaling pathway," Current Biology, vol. 16, no. 21, pp. 2081-2089, 2006.

[55] M. Willecke, F. Hamaratoglu, M. Kango-Singh et al., "The fat cadherin acts through the hippo tumor-suppressor pathway to regulate tissue size," Current Biology, vol. 16, no. 21, pp. 2090-2100, 2006.

[56] M. Overholtzer, J. Zhang, G. A. Smolen et al., "Transforming properties of YAP, a candidate oncogene on the chromosome 11q22 amplicon," Proceedings of the National Academy of Sciences of the United States of America, vol. 103, no. 33, pp. 12405-12410, 2006.

[57] J. Dong, G. Feldmann, J. Huang et al., "Elucidation of a universal size-control mechanism in Drosophila and mammals," Cell, vol. 130, no. 6, pp. 1120-1133, 2007.

[58] F. D. Camargo, S. Gokhale, J. B. Johnnidis et al., "YAP1 increases organ size and expands undifferentiated progenitor cells," Current Biology, vol. 17, no. 23, pp. 2054-2060, 2007.

[59] M. A. St John, W. Tao, X. Fei et al., "Mice deficient of Lats1 develop soft-tissue sarcomas, ovarian tumours and pituitary dysfunction," Nature Genetics, vol. 21, no. 2, pp. 182-186, 1999.

[60] J. H. Lee, T. S. Kim, T. H. Yang et al., "A crucial role of WW45 in developing epithelial tissues in the mouse," The EMBO Journal, vol. 27, no. 8, pp. 1231-1242, 2008.

[61] N. Tapon, K. F. Harvey, D. W. Bell et al., "salvador Promotes both cell cycle exit and apoptosis in Drosophila and is mutated in human cancer cell lines," Cell, vol. 110, no. 4, pp. 467-478, 2002.

[62] Z. C. Lai, X. Wei, T. Shimizu et al., "Control of cell proliferation and apoptosis by mob as tumor suppressor, mats," Cell, vol. 120, no. 5, pp. 675-685, 2005.

[63] C. Ling, Y. Zheng, F. Yin et al., "The apical transmembrane protein Crumbs functions as a tumor suppressor that regulates Hippo signaling by binding to Expanded," Proceedings of the National Academy of Sciences of the United States of America, vol. 107, no. 23, pp. 10532-10537, 2010.

[64] C. L. Chen, K. M. Gajewski, F. Hamaratoglu et al., "The apical-basal cell polarity determinant Crumbs regulates Hippo signaling in Drosophila," Proceedings of the National Academy of Sciences of the United States of America, vol. 107, no. 36, pp. 15810-15815, 2010.

[65] E. C. Richardson and F. Pichaud, "Crumbs is required to achieve proper organ size control during Drosophila head development," Development, vol. 137, no. 4, pp. 641-650, 2010.
[66] B. S. Robinson, J. Huang, Y. Hong, and K. H. Moberg, "Crumbs regulates Salvador/Warts/Hippo signaling in Drosophila via the FERM-domain protein Expanded," Current Biology, vol. 20, no. 7, pp. 582-590, 2010.

[67] H. Lu and D. Bilder, "Endocytic control of epithelial polarity and proliferation in Drosophila," Nature Cell Biology, vol. 7, no. 12, pp. 1132-1139, 2005.

[68] N. A. Grzeschik, L. M. Parsons, M. L. Allott, K. F. Harvey, and H. E. Richardson, "Lgl, aPKC, and Crumbs regulate the Salvador/Warts/Hippo pathway through two distinct mechanisms," Current Biology, vol. 20, no. 7, pp. 573-581, 2010.

[69] N. Nishioka, K. I. Inoue, K. Adachi et al., "The Hippo signaling pathway components Lats and Yap pattern Tead4 activity to distinguish mouse trophectoderm from inner cell mass," Developmental Cell, vol. 16, no. 3, pp. 398-410, 2009.

[70] S. Tao and K. Sampath, "Alternative splicing of SMADs in differentiation and tissue homeostasis," Development Growth and Differentiation, vol. 52, no. 4, pp. 335-342, 2010.

[71] X. Varelas, P. Samavarchi-Tehrani, M. Narimatsu et al., "The Crumbs complex couples cell density sensing to Hippo-dependent control of the TGF-beta-SMAD pathway," Developmental Cell, vol. 19, no. 6, pp. 831-844, 2010.

[72] R. Kopan and M. X. Ilagan, "The canonical Notch signaling pathway: unfolding the activation mechanism," Cell, vol. 137, no. 2, pp. 216-233, 2009.

[73] S. Guo, M. Liu, and R. R. Gonzalez-Perez, "Role of Notch and its oncogenic signaling crosstalk in breast cancer," Biochimica et Biophysica Acta-Reviews on Cancer, vol. 1815, no. 2, pp. 197-213, 2011.

[74] H. Herranz, E. Stamataki, F. Feiguin, and M. Milán, "Selfrefinement of Notch activity through the transmembrane protein Crumbs: modulation of gamma-secretase activity," The EMBO Reports, vol. 7, no. 3, pp. 297-302, 2006.

[75] K. M. Klueg and M. A. Muskavitch, "Ligand-receptor interactions and trans-endocytosis of Delta, Serrate and Notch: members of the Notch signalling pathway in Drosophila," Journal of Cell Science, vol. 112, part 19, pp. 3289-3297, 1999.

[76] A. L. Parks, K. M. Klueg, J. R. Stout, and M. A. Muskavitch, "Ligand endocytosis drives receptor dissociation and activation in the Notch pathway," Development, vol. 127, no. 7, pp. 1373-1385, 2000.

[77] Y. Mitsuishi, H. Hasegawa, A. Matsuo et al., "Human CRB2 inhibits gamma-secretase cleavage of amyloid precursor protein by binding to the presenilin complex," Journal of Biological Chemistry, vol. 285, no. 20, pp. 14920-14931, 2010.

[78] S. Ohata, R. Aoki, S. Kinoshita et al., "Dual roles of notch in regulation of apically restricted mitosis and apicobasal polarity of neuroepithelial cells," Neuron, vol. 69, no. 2, pp. 215-230, 2011.

[79] A. Genevet, C. Polesello, K. Blight et al., “The Hippo pathway regulates apical-domain size independently of its growthcontrol function," Journal of Cell Science, vol. 122, no. 14, pp. 2360-2370, 2009.

[80] C. Polesello and N. Tapon, "Salvador-warts-hippo signaling promotes Drosophila posterior follicle cell maturation downstream of notch," Current Biology, vol. 17, no. 21, pp. 1864-1870, 2007.

[81] J. Yu, J. Poulton, Y. C. Huang, and W. M. Deng, "The Hippo pathway promotes Notch signaling in regulation of cell differentiation, proliferation, and oocyte polarity," PLoS ONE, vol. 3, no. 3, article e1761, 2008. 
[82] T. J. Harris and U. Tepass, "Adherens junctions: from molecules to morphogenesis," Nature Reviews Molecular Cell Biology, vol. 11, no. 7, pp. 502-514, 2010.

[83] U. Tepass, "Crumbs, a component of the apical membrane, is required for zonula adherens formation in primary epithelia of Drosophila," Developmental Biology, vol. 177, no. 1, pp. 217-225, 1996.

[84] M. Pellikka, G. Tanentzapf, M. Pinto et al., "Crumbs, the Drosophila homologue of human CRB1/RP12, is essential for photoreceptor morphogenesis," Nature, vol. 416, no. 6877, pp. 143-149, 2002.

[85] E. Morais-de-Sá, V. Mirouse, and D. St Johnston, “aPKC phosphorylation of Bazooka defines the apical/lateral border in Drosophila epithelial cells," Cell, vol. 141, no. 3, pp. 509523,2010

[86] M. A. McGill, R. F. McKinley, and T. J. Harris, "Independent cadherin-catenin and Bazooka clusters interact to assemble adherens junctions," Journal of Cell Biology, vol. 185, no. 5, pp. 787-796, 2009.

[87] T. J. Harris and M. Peifer, "The positioning and segregation of apical cues during epithelial polarity establishment in Drosophila," Journal of Cell Biology, vol. 170, no. 5, pp. 813823, 2005.

[88] R. F. Walther and F. Pichaud, "Crumbs/DaPKC-dependent apical exclusion of Bazooka promotes photoreceptor polarity remodeling," Current Biology, vol. 20, no. 12, pp. 1065-1074, 2010.

[89] G. Berx and F. van Roy, "Involvement of members of the cadherin superfamily in cancer," Cold Spring Harbor Perspectives in Biology, vol. 1, article a003129, 2009.

[90] K. P. Harris and U. Tepass, "Cdc42 and Par proteins stabilize dynamic adherens junctions in the Drosophila neuroectoderm through regulation of apical endocytosis," Journal of Cell Biology, vol. 183, no. 6, pp. 1129-1143, 2008.

[91] J. T. Blankenship, M. T. Fuller, and J. A. Zallen, "The Drosophila homolog of the Exo84 exocyst subunit promotes apical epithelial identity," Journal of Cell Science, vol. 120, no. 17, pp. 3099-3110, 2007.

[92] S. Banerjee, A. D. Sousa, and M. A. Bhat, "Organization and function of septate junctions: an evolutionary perspective," Cell Biochemistry and Biophysics, vol. 46, no. 1, pp. 65-77, 2006.

[93] S. Tsukita, M. Furuse, and M. Itoh, "Multifunctional strands in tight junctions," Nature Reviews Molecular Cell Biology, vol. 2, no. 4, pp. 285-293, 2001.

[94] K. Turksen and T. C. Troy, "Junctions gone bad: Claudins and loss of the barrier in cancer," Biochimica et Biophysica ActaReviews on Cancer, vol. 1816, no. 1, pp. 73-79, 2011.

[95] V. C. Fogg, C. J. Liu, and B. Margolis, "Multiple regions of Crumbs3 are required for tight junction formation in MCF10A cells," Journal of Cell Science, vol. 118, no. 13, pp. 2859-2869, 2005.

[96] M. S. Balda, M. D. Garrett, and K. Matter, "The ZO1-associated Y-box factor ZONAB regulates epithelial cell proliferation and cell density," Journal of Cell Biology, vol. 160, no. 3, pp. 423-432, 2003.

[97] K. B. Hoover, S. Y. Liao, and P. J. Bryant, "Loss of the tight junction MAGUK ZO-1 in breast cancer: relationship to glandular differentiation and loss of heterozygosity," The American Journal of Pathology, vol. 153, no. 6, pp. 1767-1773, 1998.

[98] J. Kleeff, X. Shi, H. P. Bode et al., "Altered expression and localization of the tight junction protein $\mathrm{ZO}-1$ in primary and metastatic pancreatic cancer," Pancreas, vol. 23, no. 3, pp. 259-265, 2001.

[99] M. S. Balda and K. Matter, "The tight junction protein ZO1 and an interacting transcription factor regulate ErbB-2 expression," The EMBO Journal, vol. 19, no. 9, pp. 20242033,2000

[100] T. Sourisseau, A. Georgiadis, A. Tsapara et al., "Regulation of PCNA and cyclin D1 expression and epithelial morphogenesis by the ZO-1-regulated transcription factor ZONAB/DbpA," Molecular and Cellular Biology, vol. 26, no. 6, pp. 2387-2398, 2006.

[101] M. Huerta, R. Muñoz, R. Tapia et al., "Cyclin D1 is transcriptionally down-regulated by ZO-2 via an E box and the transcription factor c-Myc," Molecular Biology of the Cell, vol. 18, no. 12, pp. 4826-4836, 2007. 


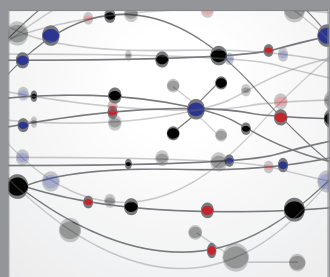

The Scientific World Journal
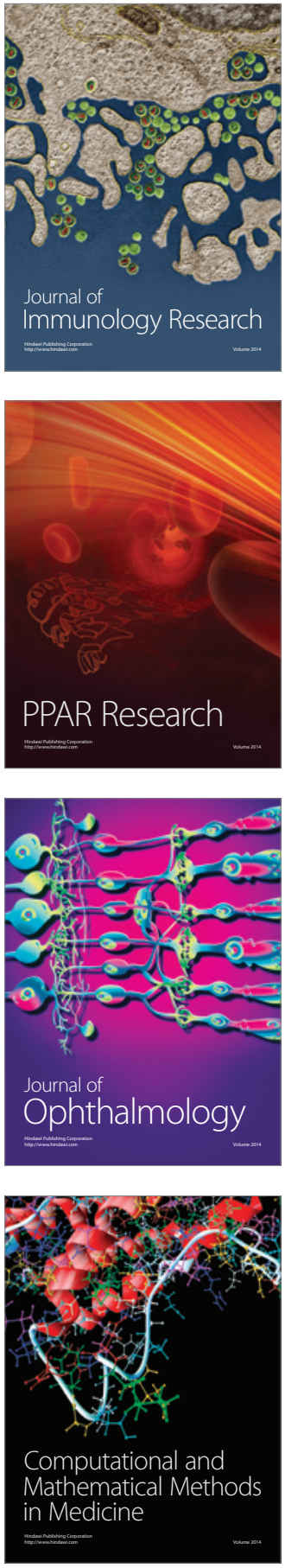

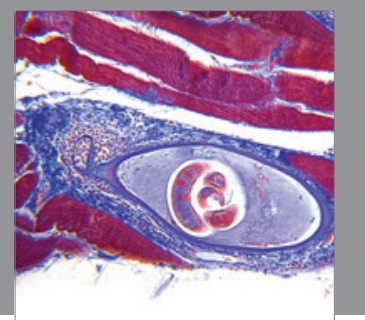

Gastroenterology

Research and Practice
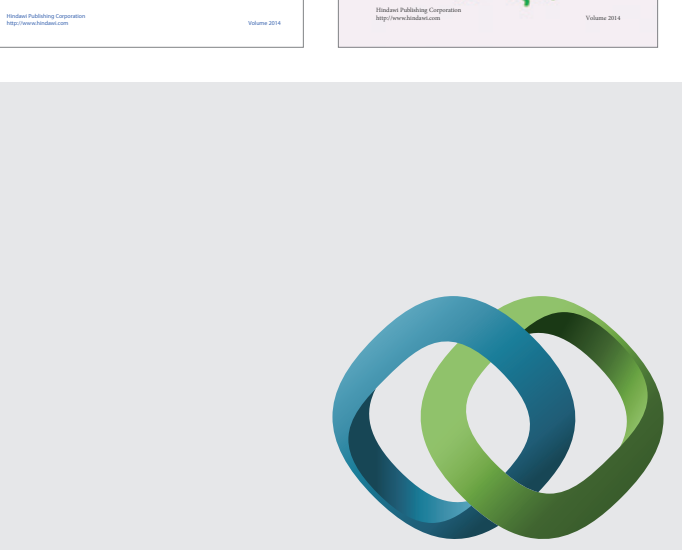

\section{Hindawi}

Submit your manuscripts at

http://www.hindawi.com
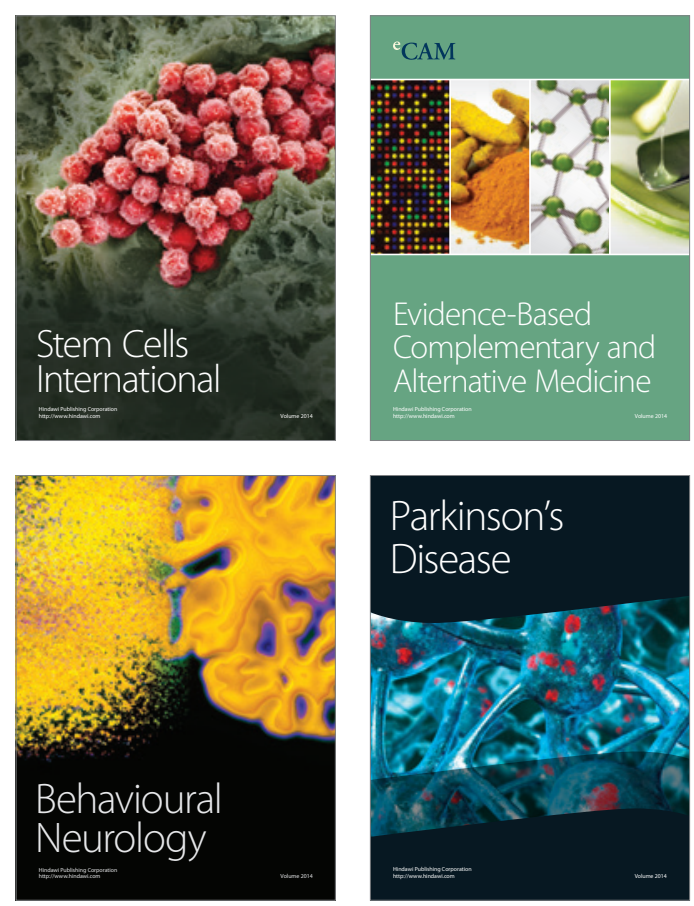

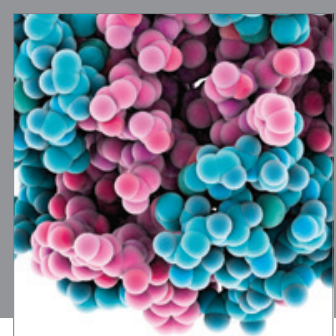

Journal of
Diabetes Research

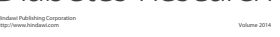

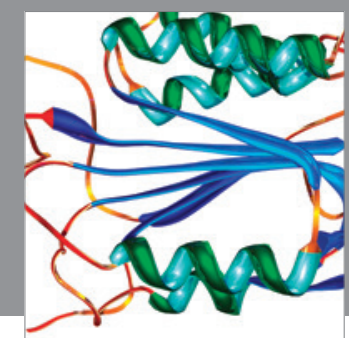

Disease Markers
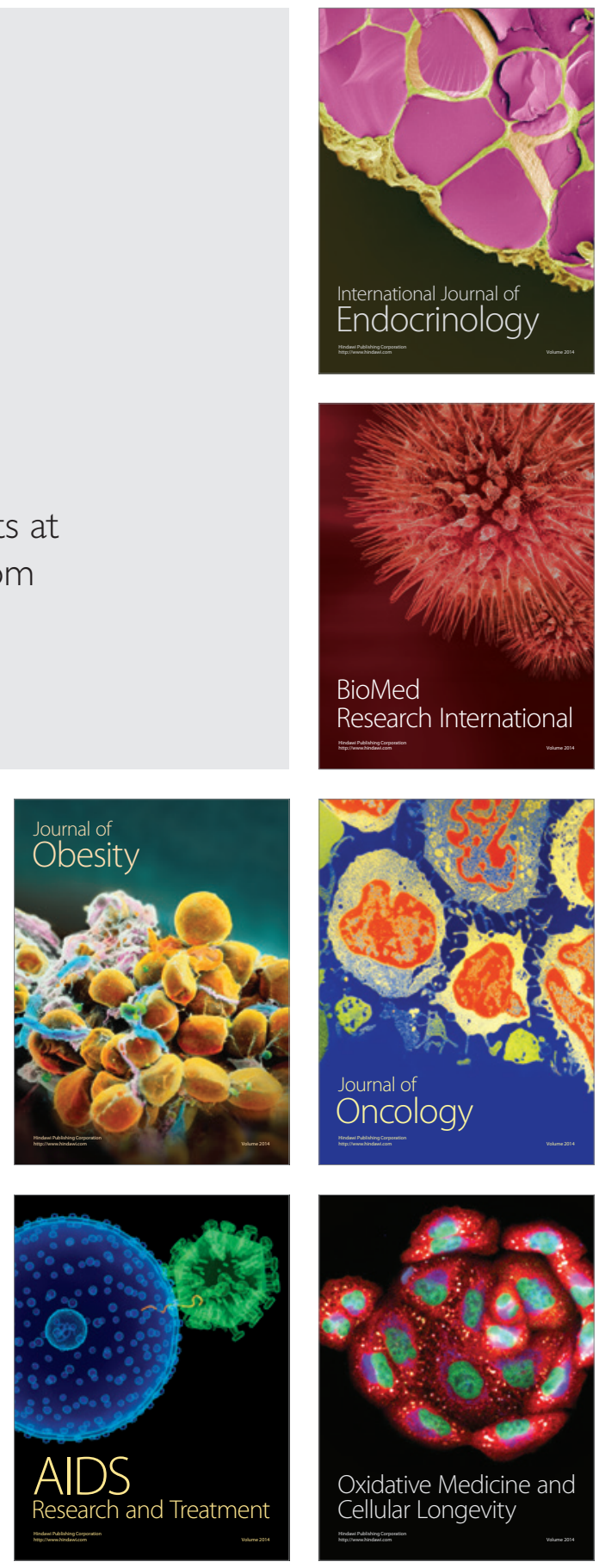